\title{
Introducción. Los cuidados en la encrucijada del cambio social
}

\author{
Introduction. Care at the crossroads of social change
}

Antía Pérez-Caramés

Recibido: 09/12/2021

Aceptado: 22/12/2021

\section{RESUMEN}

Este artículo contiene una introducción al número monográfico de Atlánticas. Revista Internacional de Estudios Feministas en torno a los estudios de cuidados que lleva por título "Los cuidados en la encrucijada del cambio social". En primer lugar, se presenta una contextualización en la que se resalta la relevancia de los cuidados para los estudios feministas y de género. A continuación, se emprende una revisión y síntesis de las principales temáticas que ha alumbrado el concepto de cuidados, desde la ciudadanía y el Estado de Bienestar, hasta las formas de organización social y reparto de los trabajos o las políticas públicas en materia de cuidados; pasando por cuestiones emergentes tales como la participación de los hombres en estos trabajos, la relación entre urbanismo y cuidados o las implicaciones de la tecnología para los cuidados. Por último, se indica y contextualiza la relevancia y originalidad de las contribuciones contenidas en el monográfico.

Palabras clave: cuidados, ciudadanía, reproducción, bienestar, sostenibilidad de la vida.

\begin{abstract}
This article presents an introduction to the special issue of Atlánticas. Revista Internacional de Estudios Feministas on care studies entitled "Care at the crossroads of social change". First, a contextualization is presented that highlights the relevance of care for feminist and gender studies. Next, a review and synthesis of the main topics that the concept of care has shed light on is undertaken, from citizenship to the Welfare State, to the forms of social organization and distribution of work or public policies in care issues; going through emerging issues such as the participation of men in this work, the relationship between urban planning and care, or the implications of technology for care. Finally, the relevance and originality of the contributions present in this special issue is indicated and contextualized.
\end{abstract}

Keywords: care, citizenship, reproduction, welfare, sustainability of life.

Antía Pérez-Caramés es Profesora Contratada Doctora en el Departamento de Sociología y Ciencias de la Comunicación de la Universidade da Coruña, donde integra el Equipo de Investigación Sociedades en Movimiento (ESOMI) y el Centro de Estudos de Xénero e Feministas (CEXEF). Correo electrónico: antia.perez@udc.es. ID: https: / / orcid.org/0000-0002-6295-0613

Cómo citar este artículo: Pérez-Caramés, Antía (2020). Introducción. Los cuidados en la encrucijada del cambio social. Atlánticas. Revista Internacional de Estudios Feministas, 5 (1), 1-29. doi: http:/ / dx.doi.org/10.17979/ arief.2020.5.1.8823 


\section{INTRODUCCIÓN. LOS CUIDADOS EN EL NÚCLEO DEL PENSAMIENTO FEMINISTA}

En el momento en que escribo esta introducción estamos ya por la sexta ola de una pandemia que se prolonga por casi dos años y que, entre muchas otras implicaciones, ha supuesto la emergencia en el debate público de la cuestión de los cuidados; de las formas en que estos se organizan socialmente y de cómo esta organización se entrelaza con los sistemas de estratificación social, sexual y racial vigentes en nuestra sociedad hasta el punto de alcanzar una situación crítica. En este sentido, la expansión mundial del coronavirus ha tenido un papel tremendamente clarividente a la hora de poner de relieve situaciones de enorme desigualdad en el acceso a servicios y prestaciones de cuidados, pero también condiciones profundamente injustas e indignas en su distribución y ejercicio por parte de quienes se dedican a su provisión. Esto es, la epidemia ha sacado a la luz la existencia de una crisis de reproducción de escala planetaria de la que los estudios feministas y de género venían ya hablando desde hace décadas.

En efecto, si la discusión en torno a los cuidados parece haberse hecho ubicua en los debates políticos y sociales más recientes, no deja de ser también cierto que es una de las cuestiones que conforman la espina dorsal de las preocupaciones del pensamiento feminista y de las pioneras de las ciencias sociales desde sus comienzos. Así, si hacemos un repaso somero de los principales puntos de interés de las primeras científicas sociales comprobaremos cómo la cuestión del trabajo doméstico ha permeado sus inquietudes intelectuales, tal y como se ha indicado en algunas obras de referencia al respecto (McDonald, 1994; Madden, Pujol y Seiz, 2004; Lengermann y Niebrugge, 2019) Esto resulta innegable al analizar la obra de Charlotte Perkins Gilman, pionera de la economía feminista, en la que el abordaje del trabajo doméstico resulta central para la comprensión de la estratificación de género, y que se refleja, principalmente en dos de sus obras: "Women and economics" (2008 [1898]) y "The Home" (1903). En diálogo con el trabajo de Gilman y su análisis sobre las condiciones de opresión de las mujeres en el hogar y el trabajo, aunque con una alternativa más próxima a la defensa del salario para el ama de casa, produce su pensamiento al otro lado del charco Marianne Weber (Ritzer, 2011; García-Sainz, 2021). Los trabajos de 
Ida Wells-Barnett y Harriet Martineau se acercan al análisis de los cuidados a partir del estudio del servicio doméstico y, particularmente, de la intersección entre sexo, raza y clase en la producción de desigualdades. Este trabajo será continuado por otras pioneras del pensamiento sociológico como Grace Abbot, que lo enfocará hacia el trabajo doméstico desempeñado por mujeres migrantes en la ciudad de Chicago (1908), convirtiéndose así en una de las pioneras en el análisis del papel de las mujeres migrantes en la reproducción social.

No obstante, no será hasta los años setenta, con la tercera ola del feminismo, y particularmente en el marco del feminismo radical, cuando esta cuestión devenga central a la teorización feminista, de mano del giro hacia "lo personal es político" (Hanisch, 1970) y de la consideración del patriarcado como sistema de dominación también en lo económico. El denominado “debate sobre el trabajo doméstico" ${ }^{\prime \prime}$ ocupará buena parte de la agenda temática de esta tercera ola. Bajo la influencia del feminismo de corte marxista se desarrolla un interés por comprender el papel que juega el trabajo doméstico en el orden capitalista. De mano de feministas italianas como Dalla Costa (1972), Fortunati (1995) [1981], Federici (2018) o Picchio (2011) [1981] se avanza el concepto de reproducción social para dar cuenta del proceso de creación de la domesticidad que viene de mano de la emergencia del capitalismo y que está en el origen tanto de un profundo cambio en las formas familiares, como de una particular relación entre el trabajo remunerado y no remunerado. Así, y siguiendo a Borderías, Carrasco y Torns (2011: 31), la reproducción social “se entendería (...) como un complejo proceso de tareas, trabajos y energías, cuyo objetivo sería la reproducción de la población y de las relaciones sociales y, en particular, la reproducción de la fuerza de trabajo (...); proceso que determinaría la posición de las personas dentro del mercado laboral al sentar las bases de los estándares de vida de la población y estructurar las relaciones de clase y la distribución de la renta". En el marco de este debate se desarrollaron temáticas que adquirieron relevancia e individualidad en las décadas siguientes, siendo algunas de las más significativas la cuestión del carácter de los cuidados como trabajo y el papel de las emociones y la

\footnotetext{
- Entre las obras de referencia que sintetizan los principales aspectos de este debate se encuentran las de Molyneux (1994); Miles (1983); Vogel (2000) En castellano se puede consultar la magnífica recopilación a cargo de Dinah Rodríguez y Jennifer Cooper (2005).
} 
relacionalidad en el mismo, la responsabilidad del Estado en la provisión de cuidados y la remuneración de los mismos, la naturaleza de la relación entre el trabajo de reproducción y la economía capitalista (concretamente, su carácter estructural), entre otros.

El punto final de este debate es, a juicio de algunas, un cierto fracaso por la incapacidad del molde teórico marxista para dar cuenta por sí solo de la raíz de la opresión de las mujeres (Himmelweit, 1987), si bien también hay quien defiende, como Lourdes Benería (1999) que la discusión se ha cerrado en falso. De las cenizas de este debate ha emergido, no obstante, el fructífero y poliédrico concepto de cuidados, que ha permitido, además, en buena parte de las Ciencias Sociales, pero particularmente en la Sociología y la Economía, el desplazamiento del eje de la atención de lo productivo a lo reproductivo, en una reivindicación, parte de una justicia social global, de la centralidad de los cuidados y de la intrínseca interdependencia y vulnerabilidad humanas (Williams, 2018).

En este ámbito surge también la noción de sostenibilidad de la vida (Carrasco, 2001, 2016; Pérez-Orozco, 2013; entre otras) para indicar el "proceso que no solo hace referencia a la posibilidad real de que la vida continúe -en términos humanos, sociales y ecológicos-, sino a que dicho proceso signifique desarrollar condiciones de vida, estándares de vida o calidad de vida aceptables para toda la población" (Bosch et al., 2005: 322 en Carrasco, 2016: 48). En este sentido, la noción de cuidados se ha vuelto clave para la interrogación crítica y la propuesta de alternativas económicas en un contexto de recrudescencia neoliberal del capitalismo y de respuestas austericidas a sus sucesivas crisis.

Así, el estrangulamiento de los estados de bienestar europeos tras la recesión de 2008 solo ha podido ser comprendido en todas sus implicaciones y complejidad gracias a perspectivas como las propuestas desde la economía feminista y de los cuidados. Y esto es así no solo porque los recortes en derechos sociales tienen una mayor afectación en las condiciones de vida de las mujeres al ser a quienes se les asigna socialmente la responsabilidad de los cuidados en las familias, sino también porque existen otras dos pautas históricas observadas en las respuestas dadas desde las políticas públicas a las 
crisis: que la recuperación del empleo masculino acaba por ser más rápida que la del femenino y también que con las recesiones se produce también un retroceso en los avances en materia de políticas de igualdad (Gálvez Muñoz y Rodríguez Modroño, 2011; Rubery, 2014; Gálvez Muñoz, 2014).

Como apuntábamos al inicio, la Sociología de los Cuidados y, más en general, los estudios feministas en torno a los cuidados, se han vuelto también un lugar indispensable desde el que pensar las implicaciones socioeconómicas de la pandemia. La revelación del carácter esencial de estos trabajos como sostenedores de la vida en un momento en que la práctica totalidad de la esfera productiva debe parar para enfrentar la expansión del virus ha permitido que la reflexión sobre su tratamiento y consideración en nuestra sociedad se abra paso y se generalice (Moré Corral, 2020). Y tampoco debemos olvidar que la carga de trabajo (particularmente del no remunerado) que soportan las mujeres se ha incrementado durante la pandemia (Power, 2020) y que de la respuesta política que se ofrezca como salida a la crisis dependerá que las condiciones de vida de las mujeres se resientan más o menos. Se han publicado ya algunos trabajos que se interrogan cómo salir del atolladero de la crisis de los cuidados (por ejemplo, Dowling, 2021), y otros que vuelven a insistir en la idea de poner los cuidados en el centro de la economía, como el recientemente publicado "manifiesto de los cuidados" (The Care Collective, 2020). Abundan también las contribuciones que alían la crisis climática planetaria con la crisis de cuidados y que, en el marco de la corriente ecofeminista, plantean la imperiosa necesidad de su abordaje conjunto (Gottschlich y Bellina, 2017; Herrero, 2020).

En las páginas que siguen nos acercaremos, en primer lugar, a las temáticas más relevantes en el ámbito de los estudios sobre cuidados, señalando aquellos debates emergentes y más novedosos en la disciplina. De este modo, se dejará el contexto sentado para comprender las interrogaciones a las que responden las diversas contribuciones que se recogen en este monográfico. 


\section{EL ALCANCE HEURÍSTICO DE LOS CUIDADOS. PROBLEMÁTICAS CLÁSICAS Y TEMÁTICAS EMERGENTES}

En este apartado repasaremos brevemente lo que ha dado de sí en la producción teórica realizada bajo una perspectiva feminista y/o de género el concepto de cuidados, señalando, en último término aquellas temáticas que están experimentando un auge reciente por parte de la investigación social.

\subsection{Cuidados, ciudadanía y bienestar}

Quizás una de las principales virtudes del concepto de cuidados ha sido su capacidad para interrogar críticamente la noción de ciudadanía social que se esconde en la formulación de los Estados de Bienestar y que impone a las mujeres un "impuesto reproductivo" en forma de explotación económica y doméstica que, a juicio de Cobo (2005), se intensifica de mano del proceso de globalización. De manera sintética, podemos encontrar dos corrientes principales en este ámbito de indagación.

En primer lugar, aquella de raíz nórdica y desarrollo italiano, que, de mano de trabajos fundacionales como el de Helga Hernes (1987) o Clare Ungerson (1985, 1987), cuestionan la escasa implicación del Estado tanto en la provisión de bienestar a través del cuidado como en la eliminación de la división sexual del trabajo. Pensadoras feministas italianas enmarcadas principalmente en la rama de la Sociología de la Familia y bajo marcos interpretativos como el de la sociología de la vida cotidiana de Agnes Heller (1977), como Laura Balbo (1994) [1978] o Chiara Saraceno (1980) retoman este debate de mano de nociones como la de "doble presencia", entendida como la exigencia a las mujeres de compatibilizar su actividad en el empleo con el trabajo no remunerado en el hogar, y que ha tenido una gran aceptación en España de la mano de sociólogas como María Ángeles Durán (1986; 1987, entre otras).

Por otra parte y, partiendo de trabajos como los de Mary Daly y Jane Lewis (1998, 2000; 1992, 1997 -en el caso de esta última-) con conceptos como el de 'social care', de la crítica de Diane Sainsbury (1999) a la tipología de regímenes 
de bienestar de Esping-Andersen (1993), o del trabajo de la estadounidense Ann Shola Orloff $(1993,1996)$ sobre la consideración de ciudadanía social de las mujeres, se desarrolla una segunda línea de trabajos sobre el papel de los cuidados y los regímenes de género en los diversos modelos de estado de bienestar. Esta corriente ha ido ganando relevancia en la medida en que, de mano del avance en el envejecimiento demográfico, se ha ido consolidando la necesidad de contemplar la demanda de cuidados como un nuevo riesgo social a abordar mediante políticas de cuidados de larga duración por parte de los estados de bienestar, particularmente de aquellos más protectores.

\subsection{La respuesta global a la crisis de los cuidados}

De hecho, una de las propuestas conceptuales que se ha adelantado, de mano de la economía feminista, para caracterizar la situación de muchos países envejecidos del norte global es la de "crisis de los cuidados" (Benería, 2008; Ezquerra, 2011; entre otras). En este ámbito, una de las intersecciones académicas más fértiles es la que han conjugado los estudios feministas y de género con los análisis sobre el fenómeno migratorio en torno a los cuidados. La intensificación del recurso a la solución global para hacer frente a la crisis de los cuidados es una de las razones que explica este auge. Este análisis macroestructural de la circulación global y desigual de los trabajos de cuidados entre diversas regiones del mundo ha sido puesto de relieve por trabajos como los de Bridget Anderson (2000), Barbara Ehrenreich y Arlie Russell Hochschild (2002) o Rhazel Salazar Parreñas (2001); pero también se han producido contribuciones al respecto bajo el paradigma de la reproducción social (Bakker y Gill, 2003; Mezzadri, Newman y Stevano, 2021).

La propuesta del concepto de cadenas globales de cuidados, para dar cuenta del particular modo en que se imbrican los países del sur y del norte global en las transferencias de trabajos de cuidados de manos de mujeres autóctonas a mujeres migrantes (Hochschild, 2000) se ha demostrado como una importante contribución para el análisis del modo concreto en que se articula la reproducción social globalizada y las implicaciones que conlleva en materia de desigualdad. No obstante, este enfoque refuerza una perspectiva que, bajo el prisma del intercambio desigual de cuidados entre unos y otros países, lo que se ha llamado el enfoque del 'care drain' y 'care gain' (Lutz y Palenga- 
Möllenbeck, 2012), oscurece el hecho de que los cuidados circulan a través del espacio transnacional y que también pueden prestarse a distancia, tal y como han destacado, entre otras, las investigaciones de Baldassar y Merla (2014).

La interrogación sobre el papel de las mujeres migrantes en la provisión de cuidados ha sido especialmente relevante en el contexto de los estados de bienestar consolidados. En efecto, numerosos países del ámbito europeo vieron emerger en pocos años un actor en el mapa de la protección social, las mujeres migrantes, ocupando un hueco, el de la respuesta a las demandas crecientes de cuidados, que había sido práctica o insuficientemente desatendido por las políticas sociales hasta ese momento. Así, cobra una relevancia especial en este contexto el análisis de la intersección entre los regímenes de bienestar, género y migraciones en los diversos modelos de bienestar (Kofman, 2008; Kilkey, Lutz y Palenga-Möllenbeck, 2010), pero también emerge el interés por dar cuenta de una de las particularidades del régimen de cuidados en los países el sur de Europa: la participación de las mujeres migrantes como empleadas de hogar (Bettio, Simonazzi y Villa, 2006; Hobson, Hellgren y Serrano, 2018). El papel central que juegan el empleo de hogar en regímenes de cuidados como el español es una particularidad que ha sido destacada en numerosos trabajos (Martínez Buján, 2011; Agrela Romero, 2011; Climent, 2011).

\subsection{Organización social de los cuidados de larga duración: actores implicados y equilibrios en el reparto}

Hemos comentado ya cómo la cuestión de los cuidados ha sido particularmente relevante a la hora de desmontar una construcción del estado del bienestar que colocaba a las mujeres en una posición de ciudadanía social de segunda. Es precisamente bajo este marco de análisis que se articula todo un conjunto de trabajos que cuestiona la forma que, en diversas sociedades y en distintos momentos del tiempo, se ha resuelto la cuestión de los cuidados, esto es, las particulares configuraciones de los cuidados a partir del papel que juegan en ellas los diversos actores implicados: el Estado, las familias, el mercado y el ámbito comunitario (Razavi, 2007).

Si bien los debates y las investigaciones en torno a la organización social de los cuidados tienen un cierto recorrido en España, particularmente de mano de los 
estudios feministas y de género en el ámbito de las políticas de igualdad (Astelarra, 2005; Bustelo y Lombardo, 2007; Castro García, 2017; entre otras), ha sido la aprobación de la Ley 39/2006 de Promoción de la Autonomía Personal y Atención a las Personas en Situación de Dependencia (LAPAD, de aquí en adelante) la que ha propiciado un contexto en el que esta cuestión se ha abierto un hueco en la agenda público-política. De hecho, la figura de la prestación por cuidados en el entorno familiar incluida en el catálogo de servicios y prestaciones de la LAPAD y propuesta como una forma de reconocimiento hacia el trabajo "informal" desarrollado por las mujeres en los hogares al cargo de sus familiares dependientes fue significativamente contestado desde sus inicios por quienes, desde una perspectiva feminista, cuestionaban su capacidad superadora de la división sexual del trabajo de cuidados en los hogares señalando que, en todo caso, contribuía a consolidar el papel de las mujeres como cuidadoras "naturales" de sus familiares (Pérez de Orozco y Baeza Gómez, 2011; Barbadillo Griñán y Gómez García, 2011; Serrano Pascual, Artiaga Leiras y Dávila de León, 2011).

A partir de este momento, los trabajos sobre la organización social de los cuidados se multiplicaron, pudiendo identificarse varias líneas de producción de pensamiento, como las articuladas en torno a la demanda de un derecho al cuidado (Domas-d'Argemir, 2019), quienes defienden las alternativas comunitarias para hacer frente a la crisis de la reproducción (Vega Solías y Martínez Buján, 2107, Campillo y Del Olmo, 2018) y quienes proponen reformular las políticas de cuidados en el marco del estado de bienestar bajo un nuevo equilibrio entre los diversos actores implicados y un mayor compromiso público por parte del Estado (Martínez, Roldán y Sastre, 2018; Navarro y Pazos Morán, 2020; Sánchez Salmerón, Pérez Eransus y Martínez Virto, 2021). Esta última corriente tiene un particular auge, a raíz de la crisis por el Covid-19, con el lanzamiento de una iniciativa en favor de un sistema estatal de cuidados (Herrero et al., 2020).

\subsection{Cuidados y masculinidades}


En el marco de los estudios feministas y de género ha emergido una corriente relativamente reciente de indagación sobre la masculinidad, o, más bien, sobre las masculinidades, que se ha centrado en desenmarañar las implicaciones de la construcción de la identidad masculina bajo un sistema patriarcal, al tiempo que acompañaba al desarrollo de movimientos de hombres en favor de la igualdad (Martín, 2007).

El desarrollo y auge de esta corriente de estudios ha discurrido en paralelo con la creciente concientización en torno a la necesidad de la implicación de los hombres en los trabajos domésticos y de cuidados, con vistas a una igual distribución de todos los trabajos entre mujeres y hombres que ponga fin a una de las principales fuentes de desigualdad: la división sexual del trabajo. De mano de este proceso se vienen publicando trabajos que indagan en las tendencias de cambio en la participación de los hombres en los trabajos domésticos y de cuidados en el seno de los hogares (ver, entre otros, Pearson, 2000; Hook, 2006; Treas y Drobnič, 2010).

También se ha desplegado el interés académico hacia los hombres que se ocupan en sectores laborales feminizados como los cuidados y cómo la generalización de estos procesos puede hacer resquebrajar la concepción que se tiene de ciertos empleos (Murray, 1996; Russell, 2007; Kilkey, 2010).

Por último, en la línea de aquellos trabajos que pretenden buscar las bases para la construcción de una masculinidad alternativa se encuentran aquellos que indican que la disposición e implicación de los hombres en los cuidados es una de las formas en las que debe reconstruirse esa identidad masculina no patriarcal (Hanlon, 2012; Elliot, 2016; Ruby y Scholz, 2018).

En España la reflexión sobre los hombres cuidadores se inicia en los noventa, de mano de la interrogación crítica de su papel en los hogares (Valiente Fernández, 1997) y también gracias a la difusión de los estudios críticos sobre masculinidad (Martín-Vidaña, 2021). Con la generalización de las Encuestas de Empleo del Tiempo han venido las aportaciones sobre la participación de los hombres en los trabajos de la esfera doméstica (González y Jurado Guerrero, 2009; Domínguez-Folgueras, 2012, 2015; Moreno-Colom, Ajenjo Cosp y Borràs Català, 
2018) y, en general, el debate sobre la corresponsabilidad (Torns, 2011; Moreno Mínguez, 2015).

Más recientemente se han desarrollado trabajos que indagan en su presencia en los trabajos remunerados del sector del empleo doméstico y los cuidados (Bodoque, Roca y Comas-D'Argemir, 2016; Bodoque Puerta, Soronellas y Offenhenden, 2019).

\subsection{Urbanismo y cuidados}

El interés por los cuidados ha irradiado temáticas y disciplinas que, en un primer momento, podrían parecer reacias a esta cuestión. Una de las más recientes en incorporarse han sido los estudios urbanos. De hecho, en el año 2021 se ha celebrado el Primer Congreso Internacional Feminista de Arquitectura y Cuidados² y en el año 2020 la revista Crítica Urbana publicaba un número monográfico sobre mujeres y ciudad en el que se prestaba una especial atención a la cuestión de los cuidados en muchas de sus contribuciones (Novas y Paleo, 2020; Valdivia, 2020).

La reciente aproximación del urbanismo a los cuidados ha sabido aliar la crítica a la destrucción del ecosistema con la necesidad de pensar los espacios y las ciudades colocando a los cuidados como una prioridad política del diseño urbanístico (Fitz y Krasny, 2019; Morrow y Parker, 2020).

En este ámbito son también particularmente numerosas las investigaciones sobre viviendas colaborativas de personas mayores, una iniciativa y un movimiento emergente para paliar la insuficiencia de las políticas públicas en materia de cuidados y vivienda en la vejez en España. Destaco aquí el trabajo de Christel Keller Garganté y Sandra Ezquerra Samper (2021), quienes, con base en un estudio de caso, se interrogan sobre la posibilidad de este tipo de iniciativas para una democratización de los cuidados; y también la monografía de Irati Mogollón García y Ana Fernández Cubero (2019) en torno al mismo fenómeno. El modelo que ofrecen las viviendas colaborativas es analizado también bajo el

\footnotetext{
2 La web del congreso puede consultarse en el siguiente enlace: http:/ / congresoarquitecturaycuidados.com/ (consultado el 2 de agosto de 2021).
} 
prisma del envejecimiento activo (Sánchez Medina y López de la Cruz, 2017) y de los cuidados comunitarios (Artiaga Leiras, 2021).

\subsection{Las relaciones entre tecnología y cuidados}

La discusión que incorpora el elemento tecnológico a los estudios en torno a los cuidados es también una de las temáticas emergentes en este terreno, si bien el número de publicaciones a este respecto es ya notable. En el ámbito de las Ciencias Sociales, las contribuciones orbitan entre aquellas que analizan los trabajos de cuidados en el marco del proceso de robotización del trabajo, la también denominada Cuarta Revolución Industrial, como un nicho de resistencia a la automatización del trabajo, pero también como una posibilidad quizás vacua, de consagrar menos tiempo y mejor repartido al trabajo doméstico en los hogares (Rodríguez Modroño y Addabbo, 2021), o incluso como una forma de levantar la "carga" de los cuidados de los hombros de las mujeres evitando pasar por la corresponsabilidad (Parks, 2020).

No obstante, la reflexión sobre el papel liberador de la carga reproductiva que podría jugar el avance tecnológico no es nueva, ni propia de este siglo, sino que forma parte de una agenda que parece querer promover el alcance de la igualdad por medios tecnológicos sin alterar los privilegios masculinos que se desprenden de la división sexual del trabajo. A esta conclusión ya había llegado el análisis de Betty Friedan con su mística de la feminidad (2016 [1963]) o el de Ruth Schwartz Cowan (2011 [1976]). Es decir, parafraseando a Borderías, Carrasco y Torns (2011: 24), la hipótesis de que el trabajo doméstico podría ser fácilmente taylorizable se demostró fallida.

No obstante, es indudable el crecimiento y desarrollo de los denominados "tecnocuidados", esto es, de la asistencia mediada a través de la tecnología, sin que esto implique la sustitución del cuidado material y directo, sino más bien un complemento del mismo (Gómez Bueno y Martín Palomo, 2020), por lo que es impensable hoy en día entender los cuidados alejados del mundo de las TIC. 


\subsection{El análisis de los microarreglos de cuidados}

El análisis de las formas de organización de los cuidados a nivel micro ha sido una de las líneas más fecundas en las que se han centrado los estudios sobre cuidados (Martín Palomo, 2010; Pérez-Caramés, 2010; Vázquez y Mayobre, 2015; entre otras). Sin embargo, estos trabajos han estado muy enfocados hacia determinados modelos de pareja y familia por entenderlos hegemónicos y representativos, pero esto ha supuesto un cierto descuido hacia las maneras en que estos trabajos se distribuyen en otras formas familiares. A medida que se han ramificado las investigaciones sobre cuidados, se han expandido pues los análisis que se interrogan por la forma de distribuir y repartir estos trabajos en modelos familiares no mayoritarios y diferenciando no solo entre dos esferas (privado-doméstica y público-política), sino también aludiendo al tiempo de ocio y de la participación social (Sagastizabal y Legarreta, 2016).

Así, son más recientes en España trabajos que analizan la distribución de los cuidados bajo el prisma de la diversidad familiar (Elizalde-San Miguel, 2020), contemplando, entre otros aspectos, el análisis del reparto del trabajo doméstico en parejas cohabitantes, del mismo sexo, reconstituidas, familias monomarentales, $\mathrm{u}$ hogares sin criaturas (Domínguez Folgueras, 2012; AjenjoCosp y García-Saladrigas, 2018; García-Saladrigas, 2018).

No obstante, una de las líneas más destacadas en el marco de estos estudios es la que indaga en cómo cuidan las cuidadoras. Esto es, cómo se organizan para cumplir con sus obligaciones que les asigna el mandato de género de cuidados hacia sus familiares quienes emigran a España para cuidar de otras familias (Díaz Gorfinkiel, 2008). El interrogante que se plantea es doblemente interesante, pues a escala micro y meso, permite indagar en cómo las mujeres que forman parte de hogares transnacionales desarrollan mecanismos de puesta en circulación de prácticas de cuidados que atraviesan fronteras (González Torralbo, 2013; Fuentes Gutiérrez y Agrela Romero, 2018), habiendo sido particularmente analizado el caso de la maternidad transnacional (Vives y Vázquez Silva, 2016). Pero, en el ámbito macro, ofrece claves interpretativas de calado para explicar cómo la crisis de la reproducción social se ha imbricado en 
procesos globales que implican migraciones femeninas a gran escala y el establecimiento de cadenas transnacionales de cuidados que refuerzan ejes de desigualdad de género, pero también de clase y étnico-raciales (Barañano Cid y Marchetti, 2016). De hecho, una de las líneas más prometedoras que ha surgido en este marco cuestiona los marcos de protección social de los Estados-nación a partir de las experiencias y prácticas transnacionales de atención y cuidados que ponen en práctica los hogares transnacionales (Levitt et al., 2016).

Por otra parte, no debemos olvidar que las mujeres migrantes que hoy en día están trabajando como cuidadoras en numerosos hogares españoles también envejecerán y necesitarán cuidados y que, de mantenerse el carácter transnacional de sus modelos familiares, habrá que pensar cómo hacemos frente a lo que se ha denominado "envejecimiento transnacional" (Ciobanu, Fokkema y Nedelcu, 2016; Dossa y Coe, 2017; Näre, Walsh y Baldassar, 2017; Horn y Schweppe, 2017). Ésta es precisamente una línea de investigación en alza entre aquellas que indagan en la intersección entre los cuidados y las migraciones (Escrivá, 2016; Moré, 2019).

En síntesis, la emergencia y expansión de los estudios de cuidados ha sido un proceso que ha permeado a diversas disciplinas y temáticas del ámbito de las Ciencias Sociales, pero también ha irradiado a otros campos de investigación, hasta el punto de que en hoy en día ha adquirido relevancia el cambio de enfoque que entiende el lugar preeminente de los cuidados en la sociedad y que desde ahí observa e interrogar a su alrededor.

\section{RESUMEN Y CONTRIBUCIÓN DE CADA ARTÍCULO}

En este número especial de Atlánticas. Revista Internacional de Estudios Feministas recogemos un conjunto de contribuciones a la investigación sobre el trabajo de cuidados que responde en buena medida a los retos y huecos en la literatura al respecto que hemos identificado en el apartado anterior.

El artículo de Iria Vázquez Silva, profesora de Sociología en la Universidade de Vigo, titulado “¿Cuántas piezas tiene el rompecabezas? La corresponsabilidad en el personal docente $\mathrm{d}$ investigador de la Universidad de Vigo" tiene como 
principal objetivo el análisis del modo en que el persoal docente e investigador concilia las diferentes esferas de la vida (laboral -académica-, personal y familiar), prestando una especial atención a las implicaciones que esto tiene en la trayectoria profesional. La originalidad de esta contribución reside, en primer lugar, en aliar el análisis del proceso de deriva precaria de las condiciones de trabajo en la Universidad pública con la interrogación crítica a la división sexual del trabajo en el seno de las familias con personal docente universitario. En segundo lugar, la aproximación metodológica a este objeto de investigación es inhabitual, pues se fundamenta en entrevistas de corte cualitativo, cuando lo más frecuente es emplear encuestas de usos del tiempo, por lo tanto, datos cuantitativos; de modo que se ofrece un análisis interpretativo de las consideraciones sobre los distintos tipos de trabajos y su valor. Además, su investigación arroja interesantes reflexiones sobre las implicaciones que ha tenido la actual crisis por coronavirus en la consideración y el reparto de los trabajos por sexos. Sus conclusiones apuntan directamente a la responsabilidad que instituciones públicas como la universitaria tienen a la hora de proveer condiciones de trabajo igualitarias y que favorezcan un reparto de trabajos equitativo entre hombres y mujeres, sin que se pongan trabas al progreso en las carreras académicas de las mujeres.

Helen Schwenken, profesora de Migraciones y Sociología en la Universidad de Osnabrück (Alemania) e integrante del Institute for Migration Research and Intercultural Studies (IMIS) de dicha Universidad, y Claire Hobden, directora técnica de la Oficina de Actividades Laborales de la Organización Internacional del Trabajo (OIT) y fundadora de la Research Network on Domestic Workers Rights (Red de Investigación sobre los Derechos de las Trabajadoras Domésticas ${ }^{3}$ ) firman el artículo que lleva por título "Mapping domestic workers' organizing globally". La contribución se centra en el análisis de las luchas por el reconocimiento del trabajo doméstico a nivel internacional, actualizando trabajos previos en los que mostraban las implicaciones del activismo de las trabajadoras domésticas migrantes para la política migratoria comunitaria

\footnotetext{
${ }^{3}$ Se trata de una red internacional e interdisciplinar de investigadores/as y activistas en torno al trabajo doméstico remunerado que trabajadn en colaboración con las organizaciones de las trabajadoras domésticas. Puede consultarse su web en el siguiente enlace: https://researchnetworkdomesticworkersrights.wordpress.com/
} 
(Schwenken, 2017). En ella presentan una clasificación de las formas organizativas que presentan las luchas de las trabajadoras domésticas a escala internacional en torno a tres tipos: el modelo del sindicato, el modelo asociativo y el modelo híbrido (que se caracteriza por una forma asociativa de las trabajadoras domésticas que se relaciona o incluso se integra en sindicatos para obtener representación, servicios y contacto con otros/as trabajadores/as). Realizan un análisis de la evolución reciente de esta tipología organizativa para concluir que en la actualidad existe una tendencia hacia la forma sindical, lo que, a juicio de las autoras, se debe principalmente al influjo de haber conseguido, en el año 2011, que la OIT promoviese el convenio 189 por un trabajo decente para las trabajadoras domésticas, lo que facilitó un mayor entendimiento entre las organizaciones de las trabajadoras domésticas y los sindicatos.

El trabajo de Marina Sagastizabal, profesora de Sociología en la Universidad del País Vasco y experta en análisis de usos del tiempo desde una perspectiva feminista, lleva por título "Pensando en otros horizontes posibles: una reflexión feminista a partir de la ciudadanía, el tiempo y los cuidados", y presenta un desafío teórico al modelo de ciudadanía que sirve de molde para buena parte de nuestros Estados de Bienestar y que se fundamenta en su sesgo marcadamente androcéntrico y, por ende, productivista. El hecho de que la ciudadanía social, y los derechos que de ella derivan, giren en torno a la órbita productiva de la economía supone la invisibilización y desvalorización de los trabajos doméstico y de cuidados, que son los que se ha asignado histórica y socialmente a las mujeres. Bajo una perspectiva feminista y alumbrada por el prisma de la sostenibilidad de la vida, la autora realiza una reinterpretación en clave histórico-temporal del concepto de ciudadanía, con el propósito de avanzar en un concepto de 'ciudadanía inclusiva', aunque haciendo también referencia a otros modelos, como el de la 'cuidadanía' o el de la 'ciudadanía sostenible' o 'ciudadanía ecológica'. Esta indagación crítica supone también un intento de superar el marco de análisis establecido por las políticas de conciliación, con vistas a caminar hacia la consideración del 'tiempo de vida', que suponga un reparto equitativo de los tiempos productivos y reproductivos en la vida cotidiana. 
La contribución de la profesora de Sociología en la Universidade da Coruña e integrante del Equipo de investigación Sociedades en Movimiento (ESOMI), Paloma Moré Corral, con el título “Más allá del empleo: la centralidad del trabajo de cuidados en el curso de vida de las mujeres migrantes", recoge un análisis sobre el papel y la relevancia del trabajo de cuidados en las trayectorias vitales de las mujeres migrantes en España. La fundamentación empírica de su trabajo reside en un enfoque de corte cualitativo, con base en entrevistas en profundidad con mujeres migrantes que desempeñan (o han desempeñado) trabajos en el sector de los cuidados a personas mayores. De su trabajo destaca la novedad y originalidad de incorporar un enfoque de curso de vida para dar cuenta de las trayectorias de las mujeres migrantes, colocando en el centro del análisis a los cuidados. De hecho, su principal conclusión subraya el lastre que supone para las trayectorias de las mujeres migrantes el haberse dedicado a trabajos en el ámbito de los cuidados, por encontrarse estos entre los peor remunerados del mercado laboral y porque además esto suele conllevar una restricción de las oportunidades laborales al margen de este sector. Por otra parte, dada la participación de estas mujeres en cadenas transnacionales de cuidados establecidas entre España y su país de origen (Ecuador, en este caso), las características del empleo de hogar acaban suponiendo una dificultad añadida para ejercer el cuidado de sus familiares y disfrutar de la vida en familia.

En torno a las relaciones entre cuidados y tecnología gira la contribución de María Teresa Martín Palomo, profesora de Sociología en la Universidad de Almería e integrante del Centro de Estudio de las Migraciones y las Relaciones Interculturales (CEMyRI) de la misma Universidad, y Carmuca Gómez Bueno, profesora de Sociología en la Universidad de Granada, titulada “Geometría fractal del cuidado: potencialidades de las tecnologías para unas políticas del cuidado de calidad en el ámbito domiciliario". En ella recogen los resultados de una investigación en torno al papel de la tecnología en la mejora de los programas que satisfacen las demandas de cuidados de las personas en situación de dependencia, centrándose particularmente en el servicio de ayuda a domicilio. Su investigación cuenta con una dimensión aplicada, pues está encaminada a implementar una herramienta tecnológica que optimice el 
servicio de ayuda a domicilio, contando, para su diseño, con la opinión al respecto tanto de las personas usuarias, como de sus familiares y las trabajadoras (auxiliares). Además de esta orientación a la resolución de un problema concreto de investigación, la contribución contiene también una reflexión de actualidad y calado sobre la relación entre tecnología y cuidados. Y es que, como las propias autoras destacan, existen un gran potencial reflexivo y analítico en la imbricación entre los estudios que se realizan desde las Ciencias Sociales en materia de ciencia y tecnología con aquellos que se desarrollan en torno a los cuidados y este artículo es una excelente muestra de ello.

El monográfico cierra con una entrevista a Silvia Federici, Profesora Emérita de Historia en la Hofstra University de Nueva York, activista feminista y escritora que cuenta con una dilatada y reconocida trayectoria en el terreno de los estudios sobre la reproducción social y la defensa de los derechos de las trabajadoras del hogar.

\section{Agradecimientos}

El trabajo de revisión teórico-empírica que se recoge en este artículo introductorio se ha desarrollado en el marco del proyecto de I+D+I "Crisis, dinámicas migratorias y condiciones de vida de la población migrante en España. Análisis comparado de los efectos de la Gran Recesión y la Gran Pandemia" (PID2020-118716RB-I00), dirigido por Antía Pérez-Caramés y financiado por el Ministerio de Ciencia e Innovación. 


\section{BIBLIOGRAFÍA}

Basaglia, Franca (1983). Mujer, locura y sociedad. Puebla: Benemérita Universidad Autónoma.

Abbott, Grace (1908). The Chicago Employment Agency and the Immigrant Worker. American Journal of Sociology, 14 (3), 289-305.

Agrela Romero, Belén (2011). Towards a model of externalisation and denationalisation of care? The role of female migrant care workers for dependent older people in Spain. European Journal of Social Work, 15 (1), 45-61, doi: https:/ / doi.org/10.1080/13691457.2011.562009

Ajenjo-Cosp, Marc y García-Saladrigas, Núria (2018). La distribución del tiempo productivo y reproductivo en las parejas reconstituidas. ¿Son más equitativas que las biparentales? Cuadernos de Relaciones Laborales, 36 (1), 167-186, doi: https:// doi.org/10.5209/CRLA.59563

Anderson, Bridget (2000). Doing the Dirty Work? The Global Politics of Domestic Labour. Londres: Zed Books.

Artiaga Leiras, Alba (2021). Cuidados comunitarios y gobierno común de la dependencia. Las viviendas colaborativas de personas mayores. Revista $\begin{array}{lllll}\text { Española de Sociología, } & 30 & \text { (2), } & \text { 1-16, }\end{array}$ doi: https: / / doi.org/10.22325/fes/res.2021.29

Astelarra, Judith (2005). Veinte años de politicas de igualdad. Madrid: Cátedra.

Bakker, Isabella y Gill, Stephen (2003). Power, Production and Social Reproduction. Londres: Palgrave Macmillan.

Balbo, Laura (1994). La doble presencia. En Cristina Borderías y Cristina Carrasco (comps.) Las mujeres y el trabajo: rupturas conceptuales (pp. 503514). Barcelona: Icaria.

Baldassar, Loretta y Merla, Laura (2014). Transnational Familis, Migration and the Circulation of Care: Understanding Mobility and Absence in Family Life. Londres: Routledge.

Barañano Cid, Margarita y Marchetti, Sabrina (2016). Perspectivas sobre género, migraciones transnacionales y trabajo: rearticulaciones del trabajo de reproducción social y de cuidados en la Europa del Sur. Investigaciones Feministas, 7 (1), 9-33, doi: https:/ / doi.org/10.5209/rev_INFE.2016.v7.n1.53094

Barbadillo Griñán, Patricia y Gómez García, M. Victòria (2011). El cuidado como responsabilidad: Consecuencias no deseadas en la aplicación de la Ley de Dependencia. Astrolabio: revista internacional de filosofía, 11, 45-54.

Benería, Lourdes (1999). The enduring debate over unpaid labour. International Labour Review, 138 (3), 321-346, doi: https://doi.org/10.1111/j.1564913X.1999.tb00389.x 
Benería, Lourdes (2008). The crisis of care, international migration and public policy. Feminist Economics, 14 (3), 1-21, doi: https:// doi.org/10.1080/13545700802081984

Bettio, Francesca; Simonazzi, Annamaria y Villa, Paola (2006). Change in care regimes and female migration: the 'care drain' in the Mediterranean. Journal of European Social Policy, 16 (3), 271-285, doi: https:// doi.org/10.1177/0958928706065598

Bodoque Puerta, Yolanda; Soronellas, Montserrat y Offenhenden, María (2019). “Igual esto de cuidar es algo que tiene futuro”. Trayectorias laborales de hombres extranjeros en los cuidados de larga duración. AIBR: Revista de Antropología Iberoamericana, 14 (2), 299-231, doi: https:// doi.org/10.11156/aibr.v14i2.72616

Bodoque, Yolanda; Roca, Mireia y Comas-D'Argemir, Dolors (2016). Hombres en trabajos remunerados de cuidado: género, identidad labora, y cultura del trabajo. Revista Andaluza de Antropología, 11, 67-91, doi: http://dx.doi.org/10.12795/RAA.2016.11.04

Bosch, Anna; Carrasco, Cristina y Grau, Elena (2005). Verde que te quiero violeta. Encuentros y desencuentros entre feminismo y ecologismo. En Enric Tello (ed.) La historia cuenta. Del decrecimiento económico al desarrollo sostenible (pp. 321-346). Barcelona: El Viejo Topo.

Bustelo, María y Lombardo, Emanuela (2007). Políticas de igualdad en España y en Europa. Madrid: Cátedra.

Campillo, Inés y Del Olmo, Carolina (2018). Reorganizar los cuidados. ¿Y si dejamos de hacernos las suecas? Viento Sur, 156, 77-86.

Carrasco, Cristina (2001). La sostenibilidad de la vida humana: ¿Un asunto de mujeres? Mientras Tanto, 82: 43-70.

Carrasco, Cristina (2016). Sostenibilidad de la vida y ceguera patriarcal. Una reflexión necesaria. Atlánticas - Revista Internacional de Estudios Feministas, $\quad 1 \quad$ (1), 34-57, https:// doi.org/10.17979/arief.2016.1.1.1435

Carrasco, Cristina; Borderías, Cristina y Torns, Teresa (eds.) El trabajo de cuidados. Historia, teoría y políticas. Madrid: La Catarata.

Castro García, Carmen (2017). Políticas para la igualdad. Permisos por nacimiento y transformación de roles de género. Madrid: La Catarata.

Ciobanu, Ruxandra Oana; Fokkema, Tineke y Nedelcu, Mihaela (2016). Ageing as a migrant: vulnerabilities, agency and policy implications. Journal of Ethnic and Migration Studies, 43 (2), 164-181, doi: https:// doi.org/10.1080/1369183X.2016.1238903

Climent, Susana (2011). Migrant Women and Defamilialization in the Spanish Welfare State. En Hanne Marlene Dahl, Marja Keränen y Anne Kovalainen (eds.) Europeanization, Care and Gender (pp. 152-164). Londres: Palgrave Macmillan. 
Cobo, Rosa (2005). Globalización y nuevas servidumbres de las mujeres. En Ana de Miguel Álvarez y Celia Amorós Puente (coords.) Teoría Feminista: de la Ilustración a la Globalización (pp. 265-300). Barcelona: Minerva.

Comas-d'Argemir, Dolors (2019). Cuidados y derechos. El avance hacia la democratización de los cuidados. Cuadernos de Antropología Social, 49, doi: https: / / doi.org/10.34096/ cas.i49.6190

Dalla Costa, Mariarosa y James, Selma (1972). The power of women and the subversion of the community. Londres: Butler \& Tanner.

Daly, Mary y Lewis, Jane (1998). Conceptualising Social Care in the Context of Welfare State Restructuring in Europe. Londres: Routledge.

Daly, Mary y Lewis, Jane (2000). The concept of social care and the analysis of contemporary welfare states. The British Journal of Sociology, 51 (2), 281298, doi: https: / / doi.org/10.1111/j.1468-4446.2000.00281.x

Díaz Gorfinkiel, Magdalena (2008). El mercado de trabajo de los cuidados y la creación de las cadenas globales de cuidado: ¿cómo concilian las cuidadoras? Cuadernos de Relaciones Laborales, 26 (2), 71-89.

Domínguez-Folgueras, Marta (2012). La división del trabajo doméstico en las parejas españolas. Un análisis de uso del tiempo. Revista Internacional de Sociología, 70 (1), 153-179, doi: https:/ / doi.org/10.3989/ ris.2009.08.26

Domínguez-Folgueras, Marta (2015). Parentalidad y división del trabajo doméstico en España, 2002-2010. Revista Española de Investigaciones Sociológicas, 149, 45-62, doi: http:/ / dx.doi.org/10.5477/ cis/ reis.149.45

Dossa, Parin y Coe, Cati (2017). Transnational Aging and Reconfigurations of Kin Work. Nueva York: Rutgers University Press.

Dowling, Emma (2020). The Care Crisis: What Caused It and How Can We End It. Londres: Verso.

Durán, María Ángeles (1986). La jornada interminable. Barcelona: Icaria.

Durán, María Ángeles (1987). De puertas adentro. Madrid: Instituto de la Mujer.

Ehrenreich, Barbara y Hochschild, Arlie Russell (2002). Global Woman; nanies, maids and sex workers in the new economy. Nueva York: Metropolitan Books.

Elizalde-San Miguel, Begoña (2020). La diversidad familiar ante el reto de los cuidados. Dossieres EsF, 36, 34-38.

Elliott, Karla (2016). Caring Masculinities: Theorizing and Emerging Concept. Men and Masculinities, 19 (3), 240-259, doi: https:/ / doi.org/10.1177/1097184X15576203

Escrivá, Ángeles (2016). Transforming Conceptions of Care at Home: Ageing Moroccan and Peruvian Migrants in Spain. En Katie Walsh y Lena Näre 
(eds.) Transnational Migration and Home in Older Age (pp. 201-2013). Londres: Routledge.

Esping-Andersen, Gøsta (1993) Los tres mundos del Estado del Bienestar. València: Alfons el Magnánim.

Ezquerra, Sandra (2011). Crisis de los cuidados y crisis sistémica: la reproducción como pilar de la economía llamada real. Investigaciones Feministas, 2 , 175-194, doi: https: / / doi.org/10.5209/rev_INFE.2011.v2.38610

Federici, Silvia (2018). El patriarcado del salario. Críticas feministas al marxismo. Madrid: Traficantes de sueños.

Fitz, Angelika y Krasny, Elke (2019). Critical Care Architecture and Urbanism for a Broken Planet. Cambridge, MA: Architekturzentrum Wien y The MIT Press.

Fortunati, Leopoldina (1995) [1981]. The Arcane of Reproduction. Housework, Prostitution, Labor and Capital. Nueva York: Autonomedia.

Friedan, Betty (2016) [1963]. La mística de la feminidad. Madrid: Cátedra.

Fuentes Gutiérrez, Virginia y Agrela Romero, Belén (2018). Circuitos de precariedad de las cuidadoras bolivianas en España: implicaciones familiares y supervivencias transnacionales. Migraciones internacionales, 9 (3), 121-144, doi: https: / / doi.org/10.17428/rmi.v9i34.348

Gálvez Muñoz, Lina (2014). Mujeres y crisis: un análisis feminista de la gran recesión y el austericidio. En Cristina Carrasco (ed.) Con voz propia. La economía feminista como apuesta teórica y política (pp. 193-217). Madrid: La Oveja Roja / Viento Sur.

Gálvez Muñoz, Lina y Rodríguez Modroño, Paula (2011). La desigualdad de género en las crisis económicas. Investigaciones Feministas, 2, 113-132, doi: https: / / doi.org/10.5209/ rev_INFE.2011.v2.38607

García-Sainz, Cristina (2021). Sociólogas fundadoras, la memoria oculta de la sociología. Revista Española de Sociología, 30, (2), a38, doi: https:// doi.org/10.22325/fes/res.2021.38

García-Saladrigas, Núria (2018). El uso del tiempo de los hogares reconstituidos y monoparentales. Tesis doctoral. Departamento de Geografía, Universitat Autònoma de Barcelona.

Gilman, Charlotte Perkins (2008) [1898]. Mujeres y economía: un estudio sobre la relación económica entre hombres y mujeres como factor de evolución social. València: Universitat de València.

Gómez Bueno, Carmuca y Martín Palomo, María Teresa (2020). Tecnocuidados en los hogares. Cualificaciones requeridas, activas y activables en el Servicio de Ayuda a Domicilio. Cuadernos de Relaciones Laborales, 38 (2), 231-250, doi: https: / / doi.org/10.5209/crla.70881 
González, María José y Jurado Guerrero, Teresa (2009). ¿Cuándo se implican los hombres en las tareas domésticas? Un análisis de la "Encuesta de Empleo del Tiempo". Panorama Social, 10, 65-81.

Gonzálvez Torralbo, Herminia (2013). Los cuidados en el centro de la migración. La organización social de los cuidados transnacionales desde un enfoque de género. Migraciones, 33, 127-153.

Gottschlich, Daniela y Bellina, Leonie (2017). Environmental justice and care: critical emancipatory contributions to sustainability discourse. Agriculture and Human Values, 34, 941-953, doi: https: / / doi.org/10.1007/s10460-016-9761-9

Hanisch, Carol (1970). The Personal is Political. En Shulamith Firestone (ed.) Notes from the Second Year: Women's Liberation. Major Writings of the Radical Feminists (pp. 76-77). Disponible en: https:/ / repository.duke.edu/dc/wlmpc/wlmms01039 Consultado por última vez el 10 de julio de 2021.

Hanlon, Niall (2012). Masculinities, care and equality. Identity and nurture in men's lives. Londres: Palgrave Macmillan.

Heller, Agnes (1977). Sociología de la vida cotidiana. Barcelona: Península.

Hernando, Ana (2006). Una crítica feminista a la Ley de Dependencia. Viento sur, 88: 89-95.

Hernes, Helga (1987). Welfare State and Women Power. Essays in State Feminism. Oslo: Norwegian University Press.

Herrero, Yayo (2020). Apuntes para repensar la vida en tiempos de emergencias. Libre pensamiento, 103, 53-62.

Herrero, Yayo; Morillas, Toni; Pérez Orozco, Amaia; Castro, Carmen; Campillo, Inés; Medialdea, Bibiana; Rodríguez Palop, María Eugenia; Gómez, Laura; Rodríguez, Ángela; Simó, Cristina; Meyer, Amanda; García, Nora; López Barceló, Esther; Miguela, Haizea y Alonso, Clara (2020). Aportación feminista al debate de la reconstrucción postCovid19. Hacia un sistema estatl de cuidados. Disponible en: https: / / www.congreso.es/docu/comisiones/reconstruccion/politicas sociales/comp/2_Aportacion_feminista_PO.pdf Consultado por última vez el 21 de septiembre de 2021.

Himmelweit, Susan (1987). Domestic labour. En John Eatwell; Murray Milgate y Peter Newman (eds.) The New Palgrave: A Dictionary of Economics (pp. 1-4). Nueva York: Palgrave Macmillan.

Hobson, Barbara; Hellgren, Zenia y Serrano, Inma (2018). Migrants, markets and domestic work: Do institutional contexts matter in the personal household service sector? Journal of European Social Policy, 28, 4, 386-401, https:/ / doi.org/10.1177/0958928717753578 
Hochschild, Arlie Russell (2000). Global care chains and emotional surplus value. En Will Hutton y Anthony Giddens (eds.) On the edge. Living with global capitalism (pp. 130-146). Londres: Jonathan Cape.

Hook, Jennifer, L. (2006). Care in Context: Men's Unpaid Work in 20 Countries, 1965-2003. American Sociological Review, 71 (4), 639-660, doi: https: / / doi.org/10.1177/000312240607100406

Horn, Vincent y Schweppe, Cornelia (2017). Transnational aging: toward a transnational perspective in old age research. European Journal of Ageing, 14, 335-339, doi: https: / / doi.org/10.1007/s10433-017-0446-z

Keller Garganté, Christel y Ezquerra Samper, Sandra (2021). Viviendas colaborativas de personas mayores: democratizar el cuidado en la vejez. Revista de Estudios Cooperativos, 137, 1-22, doi: https: / / doi.org/10.5209/ reve.71867

Kilkey, Majella (2010). Men and Domestic Labor: A Missing Link in the Global Care Chain. Men and Masculinities, 13 (1): 126-149, doi: https:/ / doi.org/10.1177/1097184X10382884

Kilkey, Majella; Lutz, Helma y Palenga-Möllenbeck, Ewa (2010). Introduction: Domestic and Care Work at the Intersection of Welfare, Gender and Migration Regimes: Some European Experiences. Social Policy and Society, 9 (3), 379-384, doi: https: / / doi.org/10.1017/S1474746410000096

Kofman, Eleonore (2008). Genre, migrations, reproduction sociale et Welfare state. Un état des discussions. Les Cahiers du CEDREF, 16, 101-124. doi: https:// doi.org/10.4000/ cedref.579

Lengermann, Patricia M. y Niebrugge, Gillian (2019). Fundadoras de la sociología y la teoría social 1830-1930. Madrid: CIS.

Levitt, Peggy; Viterna, Jocelyn; Mueller, Armin y Lloyd, Charlotte (2016). Transnational social protection: setting the agenda. Oxford Development Studies, $\quad 45 \quad(1), \quad 2-19, \quad$ doi: https:/ / doi.org/10.1080/13600818.2016.1239702

Lewis, Jane (1992). Gender and the Development of Welfare Regimes. Journal of European Social Policy, 2 (3), 159-173, doi: https:/ / doi.org/10.1177/095892879200200301

Lewis, Jane (1997). Gender and Welfare Regimes: Further Thoughts. Social Politics, 4 (2), 160-177, doi: https:/ / doi.org/10.1093/sp/4.2.160

Lutz, Helma; Palenga-Möllenbeck, Ewa (2012). Care Workers, Care Workers, Care Drain, and Care Chains: Reflections on Care, Migration, and Citizenship. Social Politics: International Studies in Gender, State E Society, 19 (1), 15-37, doi: https:/ / doi.org/10.1093/sp/jxr026

Madden, Kirsten; Pujol, Michèle y Seiz, Janet (2004). A Bibliography of Female Economic Though up to 1940. Londres: Routledge. 
Martín Palomo, María Teresa (2010). Los cuidados en las familias. Estudio a partir de tres generaciones de mujeres en Andalucía. Sevilla: Instituto de Estadística de Andalucía.

Martín, Sara (2007). Los estudios de la masculinidad: Una nueva mirada al hombre a partir del feminismo. En Meri Torras (ed.). Cuerpo e identidad. Estudios de género y sexualidad (pp. 89-116). Barcelona: Universitat Autònoma de Barcelona.

Martínez Buján, Raquel (2011). La reorganización de los cuidados familiares en un contexto de migración internacional. Cuadernos de Relaciones Laborales, 29

$(1)$, 93-123, doi: https:/ / doi.org/10.5209/rev_CRLA.2011.v29.n1.2

Martínez, Rosa; Roldán, Susana y Sastre, Mercedes (2018). La atención a la dependencia en España. Evaluación del sistema actual y propuesta de implantación de un sistema basado en el derecho universal de atención suficiente por parte de los servicios públicos. Estudio de su viabilidad económica y de sus impactos económicos y sociales. Papeles de Trabajo del Instituto de Estudios Fiscales 5/2018. Disponible en: https: / / www.ief.es / docs/destacados/publicaciones/papeles_trabajo/ 2018_05.pdf Consultado por última vez el 18 de septiembre de 2021.

Martín-Vidaña, David (2021). Masculinidades cuidadoras: la implicación de los hombres españoles en la provisión de los cuidados. Un estado de la cuestión. Prisma Social, 33, 228-260.

McDonald, Lynn (1994). The Women Founders of the Social Sciences. Ottawa: Carleton University Press.

Mezzadri, Alessandra; Newman, Susan y Stevano, Sara (2021). Feminist global political economies of work and social reproduction. Review of International Political Economy, doi: https:/ / doi.org/10.1080/09692290.2021.1957977

Miles, Angela (1983). Economism and Feminism: Hidden in the Household. A Comment on the Domestic Labour Debate. Studies in Political Economy, 11 (1), 197-209, doi: https: / / doi.org/10.1080/19187033.1983.11675668

Mogollón García, Irati y Fernández Cubero, Ana (2019). Arquitecturas del cuidado. Hacia un envejecimiento activista. Barcelona: Icaria.

Molyneux, Maxine (1994). Más allá del debate sobre el trabajo doméstico. En C. Carrasco Bengoa y Carme Alemany (comps.), Las mujeres y el trabajo: rupturas conceptuales (pp. 111-150). Barcelona: Icaria.

Moré Corral, Paloma (2020). Cuidados y crisis del coronavirus: el trabajo invisible que sostiene la vida. Revista Española de Sociología, 29 (3), 737745, doi: https:/ / doi.org/10.22325/fes/ res.2020.47

Moré, Paloma (2019). Hacerse mayor en el sector de los cuidados: Envejecimiento y jubilación de las mujeres ecuatorianas cuidadoras en Madrid. Sociología del Trabajo, 95, 35-51, doi: https:/ / doi.org/10.5209/stra.66443 
Moreno Mínguez, Almudena (2015). La ambivalencia ante la corresponsabilidad parental en España: una cuestión de género. La ventana. Revista de estudios de género, 5 (42): 46-98.

Moreno-Colom, Sara; Ajenjo Cosp, Marc y Borràs Català, Vicent (2019). La masculinización del tiempo dedicado al trabajo doméstico rutinario. Revista Española de Investigaciones Sociológicas, 163, 41-58, doi: http:/ / dx.doi.org/10.5477/ cis / reis.163.41

Morrow, Oona y Parker, Brenda (2020). Care, commoning and collectivity: from grand domestic revolution to urban transformation. Urban Geography, $41 \quad(4), \quad 607-624, \quad$ doi: https: / / doi.org/10.1080/02723638.2020.1785258

Murray, Susan B. (1996). "We all love Charles": Men in Child Care and the Social Construction of Gender. Gender \& Society, 10 (4), 368-385, doi: https: / / doi.org/10.1177/089124396010004002

Näre, Lena; Walsh, Katie; Baldassar, Loretta (2017). Ageing in transnational contexts: transforming everyday practices and identities in later life. Identities, $24 \quad$ (5), 515-523, doi: https: / / doi.org/10.1080/1070289X.2017.1346986

Navarro, Vicenç y Pazos Morán, María (2020). El cuarto pilar del estado del bienestar. Una propuesta para cubrir necesidades esenciales de cuidado, crear empleo y avanzar hacia la igualdad de género. Disponible en: https: / / cuartopilar.es/wp-content/uploads/2020/08/INFORME4\% C2\%BA-Pilar-del-Estado-del-Bienestar.pdf Consultado por última vez el 18 de septiembre de 2021.

Novas, María y Paleo, Natalia (2020). El feminismo y la producción de espacios para la vida. Crítica urbana, 3 (11): 4-7.

Orloff, Ann Shola (1993). Gender and the Social Rights of Citizenship: The Comparative Analysis of Gender Relations and Welfare States. American Sociological Review, 58, 303-328, doi: https: / / doi.org/10.2307/2095903

Orloff, Ann Shola (1996). Gender in the Welfare State. Annual Review of Sociology, 22: 51-78, doi: 10.1146/annurev.soc.22.1.51

Parks, Jennifer A. (2020). Lifting the Burden of Women's Care Work: Should Robots Replace the "Human Touch"? Hypatia, 25 (1): 100-120, doi: https:/ / doi.org/10.1111/j.1527-2001.2009.01086.x

Parreñas, Rhazel Salazar (2001). Servants of Globalisation: Women, Migration and Domestic Work. Stanford: Stanford University Press.

Pearson, Ruth (2000). All Change? Men, Women and Reproductive Work in the Global Economy. The European Journal of Development Research, 12, 219-237, doi: https:// doi.org/10.1080/09578810008426773

Pérez de Orozco, Amaia y Baeza Gómez, Paula (2011). Sobre "dependencia” y otros cuentos. Reflexiones en torno a la Ley de Promoción de la Autonomía Personal y Atención a las Personas en Situación de 
Dependencia. Lan Harremanak - Revista de Relaciones Laborales, 15, 13-40, doi: https:/ / doi.org/10.1387/lan-harremanak.3114

Pérez Orozco, Amaia (2013). Crisis multidimensional y sostenibilidad de la vida. Investigaciones Feministas, 1, 29-53, doi: https:/ / doi.org/10.5209/rev_INFE.2011.v2.38603

Pérez-Caramés, Antía (2010). Configuraciones del trabajo de cuidados en el entorno familiar. De la toma de decisión a la gestión del cuidado. Alternativas: Cuadernos de Trabajo Social, 17, 121-140, doi: https:// doi.org/10.14198/ ALTERN2010.17.7

Picchio, Antonella (2011) [1981]. La reproducción social y la estructura básica del mercado laboral. En Cristina Carrasco, Cristina Borderías y Teresa Torns (eds.) El trabajo de cuidados. Historia, teoría y políticas (pp. 122-144)). Madrid: La Catarata.

Power, Kate (2020). The COVID-19 pandemic has increased the care burden of women and families. Sustainability: Science, Practice and Policy, 16 (1), 6773, doi: https: / / doi.org/10.1080/15487733.2020.1776561

Razavi, Shahra (2007). The Political and Social Economy of Care in a Development Context. Gender and Development Programme Paper Number 3. Disponible en: https: / / www.unrisd.org/ 80256B3C005BCCF9 / (httpAuxPages)/2DBE6 A93350A7783C12573240036D5A0/\$file/Razavi-paper.pdf Consultado por última vez el 17 de septiembre de 2021.

Ritzer, George (2011). Las primeras sociólogas y la teoría sociológica clásica: 1830-1930. En George Ritzer (ed.) Teoría Sociológica Clásica (pp. 299-332). Madrid: McGraw Hill.

Rodríguez Modroño, Paula y Addabbo, Tindara (2021). Desigualdades en la $4^{\mathrm{a}}$ Revolución Industrial. Trabajos y cuidados en la era digital. Revista de Economía Crítica, 31, 36-43.

Rodríguez, Dinah y Cooper, Jennifer (2005) (comps.) El debate sobre el trabajo doméstico. México: Universidad Nacional Autónoma de México.

Rubery, Jill (2014). From 'women and recession' to 'women and austerity': a framework for analysis. En Maria Karamessini y Jill Rubery (eds.) Women and Austerity. The Economic Crisis and the Future for Gender Equality (pp. 17-36). Londres: Routledge.

Ruby, Sophie y Scholz, Sylka (2018). Care, care work and the struggle for a careful world from the perspective of the sociology of masculinities. Österreichische Zeitschrift für Soziologie, 43, 73-83, doi: https: / / doi.org/10.1007/s11614-018-0284-z

Russell, Richard (2007). Men Doing “Women's Work”: Elderly Men Caregivers and the Gendered Construction of Care Work. The Journal of Men's Studies, 15 (1), 1-18, doi: https:/ / doi.org/10.3149/jms.1501.1 
Sagastizabal, Marina y Legarreta, Matxalen (2016). La “triple presenciaausencia": una propuesta para el estudio del trabajo doméstico familiar, el trabajo remunerado y la participación sociopolítica. Papeles del CEIC, 151, doi: https:/ / doi.org/10.1387/pceic. 15447

Sainsbury, Diane (1999) (ed.) Gender and Welfare State Regimes. Oxford: Oxford University Press.

Sánchez Medina, José A. y López de la Cruz, Laura (eds.). Soluciones habitacionales para el envejecimiento activo: viviendas colaborativas o cohousing: respondiendo a los cambios demográficos desde la innovación social. Valencia: Tirant lo Blanch.

Sánchez Salmerón, Víctor; Pérez Eransus, Begoña y Martínez Virto, Lucía (2021). El modelo español de cuidados de larga duración ante el desafío de la desigualdad. El reto de garantizar apoyos para la población dependiente más vulnerable. Prisma Social, 32, 168-195.

Saraceno, Chiara (1980). Il laboro mal diviso. Bari: De Donato.

Schwartz Cowan, Ruth (2011) [1976]. La 'Revolución Industrial' en el hogar: tecnología doméstica y cambio social en el siglo XX. En Cristina Carrasco, Cristina Borderías y Teresa Torns (eds.) El trabajo de cuidados. Historia, teoría y politicas (pp. 97-121). Madrid: La Catarata.

Schwenken, Helen (2017). "Domestic Slavery" versus "Workers Rights": Political Mobilizations of Migrant Domestic Workers in the European Union. UC San Diego Working Papers. Disponible en: https: / / escholarship.org/content/ qt5759t7fw / qt5759t7fw.pdf Consultado por última vez el 17 de septiembre de 2021.

Serrano Pascual, Amparo; Artiaga Leiras, Alba y Dávila de León, María Celeste (2011). Crisis de los cuidados, Ley de Dependencia y confusión semántica. Revista Internacional de Sociología, 71 (3): 669-694, doi: https:// doi.org/10.3989/ ris.2012.07.30

The Care Collective (2020). The Care Manifesto. The Politics of Interdependence. Londres: Verso.

Torns, Teresa (2011). Conciliación de la vida laboral y familiar o corresponsabilidad: ¿el mismo discurso? Revista interdisciplinar de estudios de género, 1, 5-13.

Treas, Judith y Drobnič, Sonja (2010) (eds.). Dividing the Domestic. Men, Women, $\mathcal{E}$ Household Work in Cross-National Perspective. Stanford: Stanford University Press.

Ungerson, Clare (1985). Paid Work and Unpaid Caring: A Problem for the Women or the State? En Paul Close y Rosemary Collins (eds.) Family and Economy in Modern Society (pp. 146-161). Londres: Palgrave Macmillan. 
Ungerson, Clare (1987). Policy is Personal: Sex, Gender and Informal Care. Nueva York: Tavistock.

Valdivia, Blanca (2020). La penalización del cuidado en la ciudad capitalista y patriarcal. Crítica urbana, 3 (11), 15-17.

Valiente Fernández, Celia (1997). ¿Algo más que "ganadores del pan”?: El papel de los hombres en el ámbito familiar en España (1975-1996). Revista Española de Investigaciones Sociológicas, 79, 221-243.

Vázquez, Iria y Mayobre, Purificación (2015). Cuidar cuesta: un análisis del cuidado desde la perspectiva de género. Revista Española de Investigaciones Sociológicas, 151, 83-100, doi: http:/ / dx.doi.org/10.5477/ cis/ reis.151.83

Vega Solís, Cristina y Martínez Buján, Raquel (2017). Explorando el lugar de lo comunitario en los estudios de género sobre sostenibilidad, reproducción y cuidados. Quaderns-e de l'Institut Català d'Antropologia, 22 (2): 65-81.

Vives, Luna y Vázquez Silva, Iria (2016). Senegalese migration to Spain: transnational mothering practices. Journal of Ethnic and Migration Studies, 43 (3), 495-512, doi: https:// doi.org/10.1080/1369183X.2016.1186531

Vogel, Lise (2000). Domestic Labor Revisited. Science E Society, 64 (2), 151-170.

Williams, Fiona (2018). Care: Intersections of scales, inequalities and crisis. Current Sociology, 66 (4), 547-561, https:// doi.org/10.1177/0011392118765206 